\title{
Modal parameter identification from output data only
}

\author{
Joseph Lardiès ${ }^{\mathrm{a}}$ \\ Institut FEMTO-ST, DMA, 24 rue de l'Epitaphe, 25000 Besançon, France
}

\begin{abstract}
To identify the modal parameters of a vibrating system from output data only we use a state space model and usually two approaches are considered: the block Hankel matrix and its shifted version and the block observability matrix and its shifted version. It is shown in the communication that these two approaches give the same results even in the noisy data case. We present numerical and experimental results, who prove the effectiveness of the procedure.
\end{abstract}

\section{Introduction}

In modal parameter identification the key point is to determine the relationship between the system parameters and the measured data. Because in practice the system input data is often unavailable, in recent years attention has been paid to system identification when only output data is available and there are variety of available approaches to estimate structural modal parameters using output responses only. Numerous papers have been presented on system identification, acquiring the estimation of parameters from measured data. Ho and Kalman [1] introduced the minimal state space realization problem for a linear time-invariant system in which the Hankel matrix is constructed by a sequence of impulse response functions called the Markov parameters. Kung [2] proposed a concept combining singular value decomposition and minimal realization algorithm for the problem of retrieving sinusoidal processes from noisy measurements. Juang [3] introduced an eigensystem realization algorithm (ERA) for modal parameter identification and model reduction, for dynamical systems, from test data. This algorithm is an extension of the Ho-Kalman procedure where indicators are developed to quantitatively identify the system and noise modes. A stochastic subspace identification (SSI) method has been presented by Van-Overschee and De Moor [4]. The subspace method identifies the state space matrices based on the measurement and by using robust numerical techniques such as QR-factorization, singular value decomposition (SVD) and least squares. The state space matrices are related to the modal parameters and the key concept of SSI is the projection of the row space of the future outputs into the row space of the past outputs.

The fundamental problem in modal parameter identification by subspace methods is the determination of the state space matrix (or transition matrix) which characterizes the dynamics of the system. We propose in the paper two methods to estimate the transition matrix. The first method uses properties of the first block row observability matrix and the second method uses properties of the deleted first block row of the block Hankel matrix.

a e-mail: joseph.lardies@univ-fcomte.fr
It is shown that these two decomposition methods give the same modal parameters.

The paper is organized as follows: in the second section we present the two identification methods based on properties of block Hankel and observability matrices. Validity tests are presented in the third section with simulated and experimental tests in laboratory. The paper is briefly concluded in Sect. 4.

\section{Subspace identification methods}

\subsection{The discrete state space representation}

The subspace identification method assumes that the dynamic behaviour of a vibrating system can be described by a discrete time state space model [1-5]

$$
\begin{gathered}
\mathrm{z}_{k+1}=\boldsymbol{A} \mathrm{z}_{k}+w_{k} \\
y_{k}=\boldsymbol{C} \mathrm{z}_{k}+v_{k}
\end{gathered}
$$

where (1) is the state equation, (2) is the observation equation, $z_{k}$ is the unobserved state vector of dimension $n ; y_{k}$ is the $(m \times 1)$ vector of observations or measured output vector at discrete time instant $k ; w_{\mathbf{k}}$ contains the external non measured force or the excitation which can be a random force, an impulse force, a step force... and $v_{\mathbf{k}}$ is a measurement noise term. $\boldsymbol{A}$ is the $(n \times n)$ transition matrix describing the dynamics of the system and $\boldsymbol{C}$ is the $(m \times n)$ output or observation matrix, translating the internal state of the system into observations. The subspace identification problem deals with the determination of the two state space matrices $\boldsymbol{A}$ and $\boldsymbol{C}$ using output-only data $y_{k}$. The modal parameters of a vibrating system are obtained by applying the eigenvalue decomposition of the transition matrix $A$

$$
\boldsymbol{A}=\boldsymbol{\Psi} \boldsymbol{\Lambda} \boldsymbol{\Psi}^{-1}
$$

where $\boldsymbol{\Lambda}=\operatorname{diag}\left(\lambda_{i}\right), i=1,2, \ldots, n$, is the diagonal matrix containing the complex eigenvalues and $\boldsymbol{\Psi}$ contains the eigenvectors of $\boldsymbol{A}$ as columns. The eigenfrequencies $F_{i}$ 
and damping factors $\xi_{i}$ are obtained from the eigenvalues which are complex conjugate pair:

$$
\begin{gathered}
F_{\mathbf{i}}=\frac{1}{2 \pi \Delta t} \sqrt{\frac{\left[\ln \left(\lambda_{i} \lambda_{i}^{*}\right)\right]^{2}}{4}+\left[\cos ^{-1}\left(\frac{\lambda_{i}+\lambda_{i}^{*}}{2 \sqrt{\lambda_{i} \lambda_{i}^{*}}}\right)\right]^{2}} \\
\xi_{i}=\sqrt{\frac{\left[\ln \left(\lambda_{i} \lambda_{i}^{*}\right)\right]^{2}}{\left[\ln \left(\lambda_{i} \lambda_{i}^{*}\right)\right]^{2}+4\left[\cos ^{-1}\left(\frac{\lambda_{i}+\lambda_{i}^{*}}{2 \sqrt{\lambda_{i} \lambda_{i}^{*}}}\right)\right]^{2}}}
\end{gathered}
$$

with $\Delta t$ the sampling period of analyzed signals. The mode shapes evaluated at the sensor locations are the columns of the matrix $\tilde{\boldsymbol{C}}$ obtained by multiplying the output matrix $\boldsymbol{C}$ with the matrix of eigenvectors $\boldsymbol{\Psi}$ :

$$
\tilde{C}=C \Psi .
$$

We propose two methods to determine the transition matrix $\boldsymbol{A}$, in order to identify the eigenfrequencies and damping factors of a vibrating system. However we prove that these two methods are equivalent even in the case of a system with noisy data.

\subsection{Determination of the transition matrix by shifting properties}

Define the (mpx1) future data vector as $y_{k}^{+}=\left[y_{k}^{T}, y_{k+1}^{T}\right.$, $\left.\ldots, y_{k+p-1}^{T}\right]^{T}$ and the $(\operatorname{mpx} 1)$ past data vector as $y_{k-1}^{-}=$ $\left[y_{k-1}^{T}, \ldots, y_{k-p}^{T}\right]^{T}$, where the superscript $T$ denotes the transpose operation. The (mpxmp) covariance matrix between the future and the past is given by

$$
H=E\left[y_{k}^{+} y_{k}^{-T}\right]=\left[\begin{array}{cccc}
R_{1} & R_{2} & \cdot & R_{p} \\
R_{2} & R_{3} & \cdot & R_{p+1} \\
\cdot & \bullet & \cdot & \bullet \\
R_{p} & R_{p+1} & \cdot & R_{2 p-1}
\end{array}\right]
$$

where $E$ denotes the expectation operator. $\boldsymbol{H}$ is the block Hankel matrix formed with the $(m x m)$ individual theoretical auto-covariance matrices $\boldsymbol{R}_{i}=E\left[\boldsymbol{y}_{k+i} \boldsymbol{y}_{k}^{T}\right]=$ $\boldsymbol{C} \boldsymbol{A}^{i-1} \boldsymbol{G}$, with $\boldsymbol{G}=E\left[\boldsymbol{x}_{k+1} \boldsymbol{y}_{k}^{T}\right]$. In practice, the autocovariance matrices are estimated from $N$ data points and are computed by $\boldsymbol{R}_{\boldsymbol{i}}=N^{-1} \sum_{k=1}^{N} \boldsymbol{y}_{k+i} \boldsymbol{y}_{k}^{T}$. The block Hankel matrix $\boldsymbol{H}$ is written as

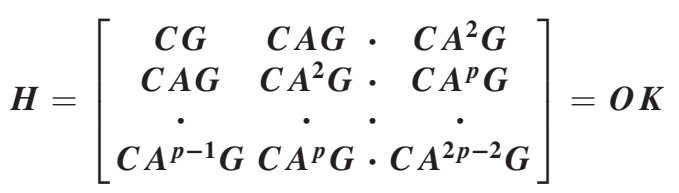

where $\boldsymbol{O}$ is the (mpxn) block observability matrix and the $\boldsymbol{K}$ is the ( $n \times \mathrm{mp})$ block controllability matrix:

$$
O=\left[\begin{array}{c}
C A \\
C A \\
\cdot \\
C A^{p-1}
\end{array}\right] ; \quad K=\left[G A G \ldots A^{p-1} G\right]
$$

The observability matrix has the form

$$
O=\left[\begin{array}{c}
O_{1} \\
C A^{p-1}
\end{array}\right]=\left[\begin{array}{c}
C \\
O_{2}
\end{array}\right]
$$

where $\boldsymbol{O}_{\mathbf{1}}$ and $\boldsymbol{O}_{\mathbf{2}}$ are $m(p-1) x n$ matrices obtained by deleting respectively the last and the first block row of the block observability matrix . It is easy to show that $\boldsymbol{O}_{2}=$ $\boldsymbol{O}_{1} \boldsymbol{A}$ and the transition matrix obtained by the deleted block row of the observability matrix method is

$$
\boldsymbol{A}_{O}=\mathrm{O}_{1}^{+} \mathrm{O}_{2}
$$

The eigenvalues of the transition matrix $\boldsymbol{A}_{\boldsymbol{O}}$ can be used to identify the modal parameters and we have

$$
\lambda\left(\boldsymbol{A}_{\boldsymbol{O}}\right)=\lambda\left(\boldsymbol{O}_{1}^{+} \boldsymbol{O}_{2}\right) .
$$

Another method to determine the transition matrix is obtained by deleting block rows of the block Hankel matrix. Let $\boldsymbol{H}_{\mathbf{1}}$ and $\boldsymbol{H}_{\mathbf{2}}$ be the matrices $m(p-1) x m p$ obtained by deleting respectively the first and the last block row of the block Hankel matrix

$$
\begin{aligned}
& H_{1}=\left[\begin{array}{cccc}
\boldsymbol{R}_{\mathbf{2}} & \boldsymbol{R}_{\mathbf{3}} & \cdot & \boldsymbol{R}_{p+1} \\
\boldsymbol{R}_{\mathbf{3}} & \boldsymbol{R}_{\mathbf{4}} & \cdot & \boldsymbol{R}_{p+2} \\
\cdot & \cdot & \cdot & \bullet \\
\boldsymbol{R}_{p} & R_{p+1} & \cdot & \boldsymbol{R}_{2 p-1}
\end{array}\right] \\
& =\left[\begin{array}{c}
C \\
C A \\
\cdot \\
C A^{p-2}
\end{array}\right] A\left[G A G \ldots A^{p-1} G\right]
\end{aligned}
$$

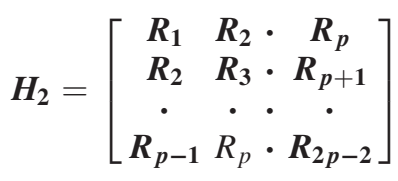

$$
\begin{aligned}
& =\left[\begin{array}{c}
C \\
C A \\
\cdot \\
C A^{p-2}
\end{array}\right] A\left[G A G \ldots A^{p-1} G\right] .
\end{aligned}
$$

From these expressions we get

$$
H_{1}=O_{1} A K=O_{2} K \text { and } H_{2}=O_{1} K
$$

and the transition matrix obtained by the shifted block row of the block Hankel matrix method is

$$
\boldsymbol{A}_{\mathrm{H}_{1}}=\mathrm{O}_{1}^{+} \mathrm{H}_{\mathbf{1}} \mathrm{K}^{+}
$$

The eigenvalues of the transition matrix $\boldsymbol{A}_{\boldsymbol{H}_{1}}$ are

$$
\begin{aligned}
\lambda\left(\boldsymbol{A}_{\boldsymbol{H}_{\mathbf{1}}}\right) & =\lambda\left(\boldsymbol{O}_{\mathbf{1}}^{+} \boldsymbol{H}_{\mathbf{1}} \boldsymbol{K}^{+}\right)=\lambda\left(\boldsymbol{H}_{\mathbf{2}}^{+} \boldsymbol{H}_{\mathbf{1}}\right)=\lambda\left(\boldsymbol{K}^{+} \boldsymbol{O}_{\mathbf{1}}^{+} \boldsymbol{O}_{\mathbf{2}} \boldsymbol{K}\right) \\
& =\lambda\left(\boldsymbol{O}_{\mathbf{1}}^{+} \boldsymbol{O}_{\mathbf{2}}\right)=\lambda\left(\boldsymbol{A}_{\boldsymbol{O}}\right) .
\end{aligned}
$$

Firstly, the eigenvalues of the transition matrix obtained by shifting properties of the block observability matrix are the same as those obtained by shifting properties 
of block rows of the block Hankel matrix and secondly the eigenvalues of the transition matrix are also the eigenvalues of the matrix $\left(\boldsymbol{H}_{2}^{+} \boldsymbol{H}_{1}\right)$. So, in practical conditions it is sufficient to form the block Hankel matrix $\boldsymbol{H}$, to extract matrices $\boldsymbol{H}_{\mathbf{1}}$ and $\boldsymbol{H}_{\mathbf{2}}$ by deleting a block row from $\boldsymbol{H}$ and compute the eigenvalues (and eventually the eigenvectors) of the matrix $\left(\boldsymbol{H}_{2}^{+} \boldsymbol{H}_{1}\right)$.

In the presence of noise, both methods use the singular value decomposition (SVD) of the block Hankel matrix to get the same performances. By retaining the $n$ dominant singular values and the corresponding sigular vectors, we get

$$
H=U S V^{T}=O K
$$

with $\boldsymbol{U}, \boldsymbol{V}$ (mpxn) matrices of singular vectors and $\boldsymbol{S}$ $(n x n)$ diagonal matrix of singular values. From (16) we choose $\boldsymbol{O}=\boldsymbol{U}$ and we consider the following matrix decomposition

$$
O=U=\left[\begin{array}{c}
U_{11} \\
U_{2}
\end{array}\right]=\left[\begin{array}{c}
U_{1} \\
U_{12}
\end{array}\right]
$$

where $\boldsymbol{U}_{\mathbf{1 1}}$ and $\boldsymbol{U}_{\mathbf{1 2}}$ are matrices formed respectively with the (p-1) first and (p-1) last block rows of the matrix of singular vectors $\boldsymbol{U}$. The eigenvalues of the transition matrix are

$$
\lambda\left(\boldsymbol{A}_{\boldsymbol{O}}\right)=\lambda\left(\boldsymbol{O}_{\mathbf{1}}^{+} \boldsymbol{O}_{\mathbf{2}}\right)=\lambda\left(\boldsymbol{U}_{\mathbf{1 1}}^{+} \boldsymbol{U}_{\mathbf{1 2}}\right)
$$

and this relation can also be obtained by shifting properties of the block Hankel matrix. Indeed, we have

$$
H_{1}=O_{2} K=U_{12} K \text { and } H_{2}=O_{1} K=U_{11} K
$$

$$
\begin{aligned}
\lambda\left(\boldsymbol{A}_{\boldsymbol{H}_{1}}\right) & =\lambda\left(\boldsymbol{H}_{\mathbf{2}}^{+} \boldsymbol{H}_{\mathbf{1}}\right)=\lambda\left(\boldsymbol{K}^{+} \boldsymbol{U}_{\mathbf{1 1}}^{+} \boldsymbol{U}_{\mathbf{1 2}} \boldsymbol{K}\right) \\
& =\lambda\left(\boldsymbol{U}_{\mathbf{1 1}}^{+} \boldsymbol{U}_{\mathbf{1 2}}\right)=\lambda\left(\boldsymbol{A}_{\boldsymbol{O}}\right) .
\end{aligned}
$$

We have showed that in the state space approach, the modal parameters obtained by shifting properties of the block Hankel matrix are the same as those obtained by shifting properties of the block observability matrix. Finally we note that the output or observation matrix $C$ can be obtained from the first block row of the matrix $\boldsymbol{O}_{1}$ or from $\boldsymbol{U}_{\mathbf{1 1}}$.

With estimates of the transition matrix $\boldsymbol{A}$ and the observation matrix $\boldsymbol{C}$ in hand we compute the eigenvalues and eigenvectors of the transition matrix and we can identify eigenfrequencies, damping factors and mode shapes of the vibrating system following (4), (6) and (6). However, all the subspace modal identification algorithms have a serious problem of model order determination. When extracting physical or structural modes, subspace algorithms always generate spurious or computational modes to account for unwanted effects such as noise, leakage, residuals, nonlinearity's ... For these reasons, the assumed number of modes, or model order, is incremented over a wide range of values and we plot the stability diagram. The stability diagram involves tracking the estimates of eigenfrequencies and damping factors as a function of model order, or assumed number of mode. As

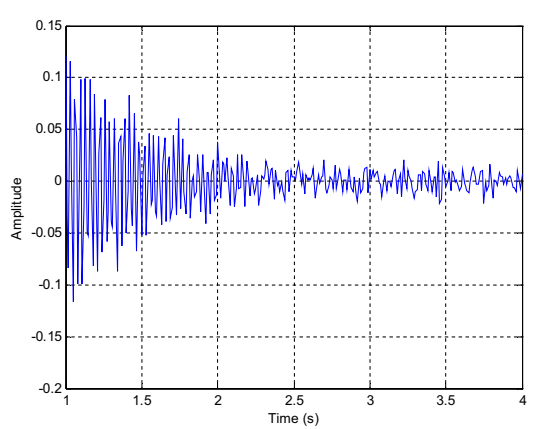

Figure 1. Time response of the simulated system.
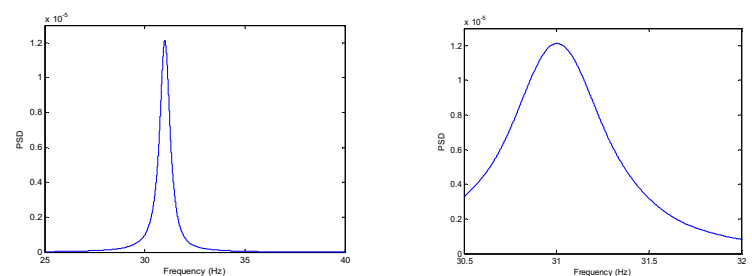

Figure 2. Spectrum for the simulated system.

the model order is increased, more and more frequencies and damping ratios are estimated, hopefully, the estimates of the physical modal parameters are stabilized using a criterion based on the modal coherence of measured modes and identified modes [5]. Using this criterion we detect and remove the spurious modes. A numerical example and two experimental tests in laboratory are now presented to identify eigenfrequencies and damping factors of vibrating systems.

\section{Applications}

\subsection{A simulated example}

To prove the effectiveness of the identification procedure based on the subspace analysis we consider a two-DOF system with very closely spaced modes. The parameters of the system are $F_{1}=30 \mathrm{~Hz}, F_{2}=30.5 \mathrm{~Hz}, \xi_{1}=0.01$ and $\xi_{2}=0.02$. Figure 1 shows the free response of the system where a Gaussian white noise has been added: the generated data were corrupted by a random noise.

The sampling frequency is $100 \mathrm{~Hz}$ and 300 time samples are used in the simulation. We convert to the frequency domain this time response by taking the discrete Fourier transform of the noisy signal. It is impossible to identify the two frequencies components by using the FFT as shown in Fig. 2, where the power spectral density has been plotted.

In our identification procedure, we plot the stabilization diagram on eigenfrequencies and damping factors. Figure 3 shows stabilization diagrams using shifting properties of the procedure presented in the previous section with the modal coherence indicator: spurious modes have been eliminated and only physical modes are present.

Our procedure can separate closely spaced modes and the mean values on identified eigenfrequencies and damping factors obtained by an average over the orders of the stabilization diagram are $\hat{F}_{1}=31 \mathrm{~Hz} ; \hat{F}_{2}=31.5 \mathrm{~Hz}$; 

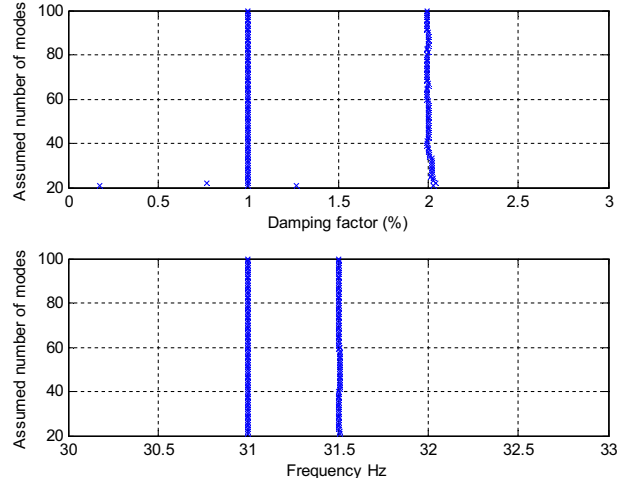

Figure 3. Stabilization diagram on eigenfrequencies and damping factors for the simulated system.
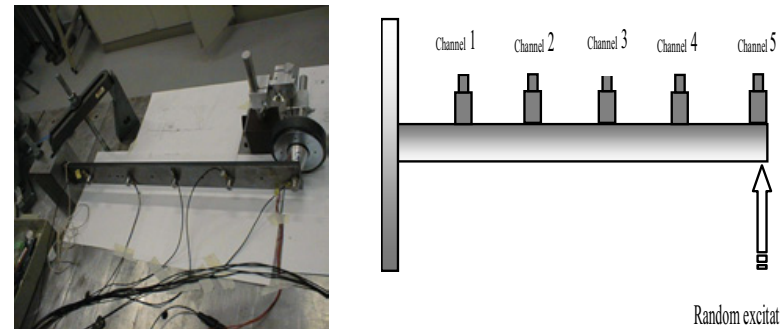

Figure 4. A clamped beam in laboratory.

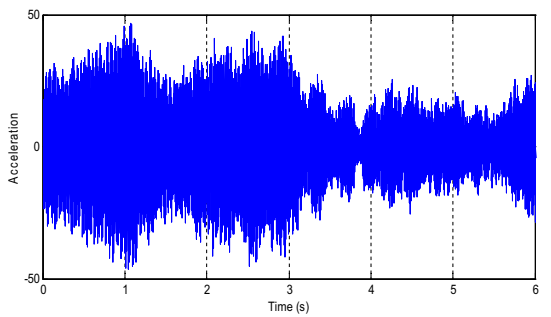

Figure 5. Time response from an accelerometer.

$\hat{\xi}_{1}=0.01 ; \hat{\xi}_{2}=0.0199$. A very satisfactory estimation on eigenfrequencies and damping factors has been obtained using simulated data. Two experimental tests in laboratory are presented in the following sections.

\subsection{Modal parameter identification of a clamped beam}

Figure 4 shows the first experimental system tested in laboratory. It is a simple clamped horizontal rectangular cantilever beam with five measurement locations equally spaced.

A Gaussian excitation is applied transversally at the free end of the beam and Fig. 5 shows a typical response of an accelerometer. Only the time responses of all accelerometers are used for modal parameter identification of the clamped beam which is excited with an unmeasured random force.

The sampling frequency of signals is $1280 \mathrm{~Hz}$ and 8192 data points are collected for each channel. Figure 6 shows the stabilization diagram on eigenfrequencies and damping factors. For comparison purposes the theoretical values on eigenfrequencies are also computed. These values are obtained from the mechanical characteristics of the beam:
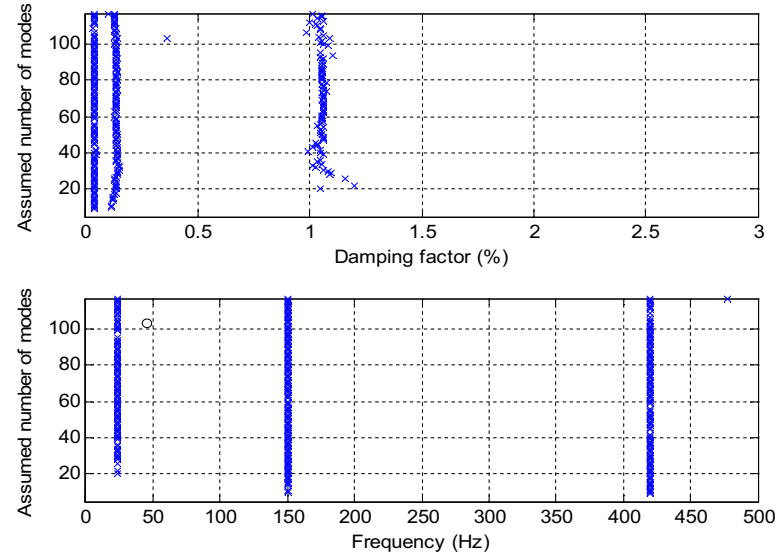

Figure 6. Stabilization diagram eigenfrequencies and damping factors for the clamped beam.

Table 1. Natural eigenfrequencies and damping factors for the experimental beam.

\begin{tabular}{|l|c|c|c|}
\hline Modes & $\begin{array}{c}\text { Theoretical } \\
\text { eigenfrequencies } \\
F_{i}(\mathrm{~Hz})\end{array}$ & $\begin{array}{c}\text { Identified } \\
\text { eigenfrequencies } \\
\hat{F}_{i}(\mathrm{~Hz})\end{array}$ & $\begin{array}{c}\text { Identified } \\
\text { damping } \\
\text { factor }(\%)\end{array}$ \\
\hline 1 & 24.13 & 24.17 & 1.06 \\
\hline 2 & 150.81 & 150.58 & 0.13 \\
\hline 3 & 422.29 & 419.72 & 0.04 \\
\hline
\end{tabular}

Young's modulus $E=2.1 \times 10^{5} \mathrm{MPa}$, mass density $\rho=$ $9000 \mathrm{~kg} \cdot \mathrm{m}^{-3}$, length of the beam $L=0,56 \mathrm{~m}$, thickness of the beam $e=97 \times 10^{-4} \mathrm{~m}$. According to the hypothesis of clamped-free beam, the flexural eigenfrequencies result from the expression derived from the Euler-Bernoulli theory:

$$
F_{i}=\frac{\alpha_{i}^{2} e}{4 \sqrt{3} \pi L^{2}} \sqrt{\frac{E}{\rho}} .
$$

Using boundary conditions of the beam we obtain the equation $\cos \left(\alpha_{i}\right) \cosh \left(\alpha_{i}\right)=-1$ and by resolution of this equation we determine the values of coefficients $\alpha_{i}$.

Table 1 gives the mean values of these identified modal parameters obtained by an average over the orders of the stabilization diagram. For comparison purposes the theoretical values on eigenfrequencies are also computed.

We note that the eigenfrequencies are very well identified; the maximum value of the relative error is only $-0.61 \%$. Another experimentation test in laboratory is presented in the next section.

\subsection{Modal parameter identification of a clamped perforated microplate}

A micro electromechanical system (MEMS) can be constituted by a perforated rectangular plate [6] as shown in Fig. 7. The main purpose of perforations is to reduce the damping and spring forces acting in the MEMS due to the fluid flow inside and around the micro structure. Generally, the modeling problem is quite complicated since the damping force acting in the moving MEMS depends on the 3D fluid flow in the perforations and also around the structure. 


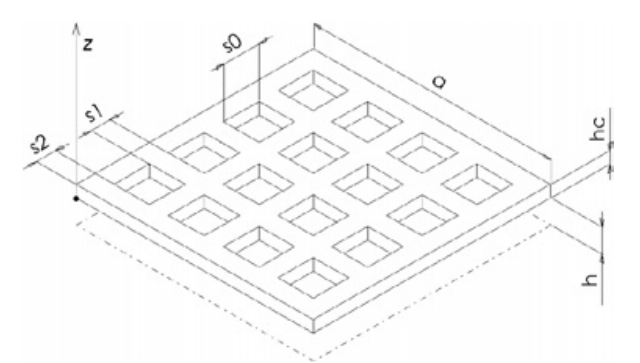

Figure 7. Schematic of the microplate.

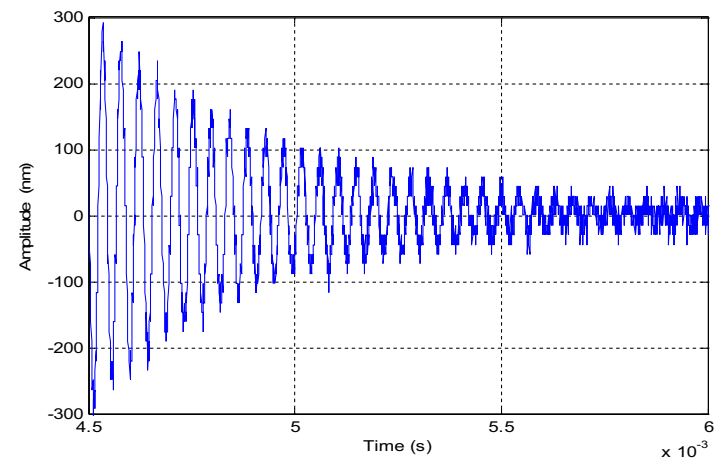

Figure 8. Time response of the microplate.

In this paper a single degree of freedom of a springmass-damper model is used to study the microplate behavior. The model is constituted by the following parameters: the plate mass $m$ concentrated in the central plate, the damping coefficient $c$ and the stiffness coefficient $k$ which are constituted of fluidic and non fluidic components. The second order differential equation describing the dynamic behavior of the microplate is:

$$
m \ddot{z}(t)+c \dot{z}(t)+k z(t)=f
$$

where $f$ is the external force acting in the microplate. In our experimental test this excitation force is a step force and Fig. 8 shows the time response of the microplate center. The dynamic measurements are conducted in the time domain by means of a laser vibrometer.

Only this time response of the structure is used in the identification procedure where the sampling frequency of signals is $2 \mathrm{MHz}$ and 3000 time samples are considered. The dimensions and material properties of the microplate are: plate side $a=185.96 \mu \mathrm{m}$, plate thickness $h_{c}=$ $6.312 \mu \mathrm{m}$, hole size $s_{0}=7.19 \mu \mathrm{m}$, plate density $\rho=$ $13920 \mathrm{~kg} \cdot \mathrm{m}^{3}$, number of holes $N_{h}=64$. The perforated microplate area is given by $A=a^{2}-s_{0} N_{h}=3.127 \times$ $10^{-8} \mathrm{~m}^{2}$ and its mass is $m=\rho A h_{c}=3.814 \times 10^{-9} \mathrm{~kg}$. The microplate stiffness is given by $k=m(2 \pi F)^{2}$ and the damping coefficient is $c=4 \pi m F \xi$ where $F$ is the resonance frequency of the perforated microplate and $\xi$ the damping factor. These two modal parameters are obtained by an average over the orders of the stabilization diagram presented in Fig. 9.

Table 2 shows the identified microplate parameters and Fig. 10 shows the comparison between the measured time response of the structure and the reconstructed response obtained from the identified modal parameters.
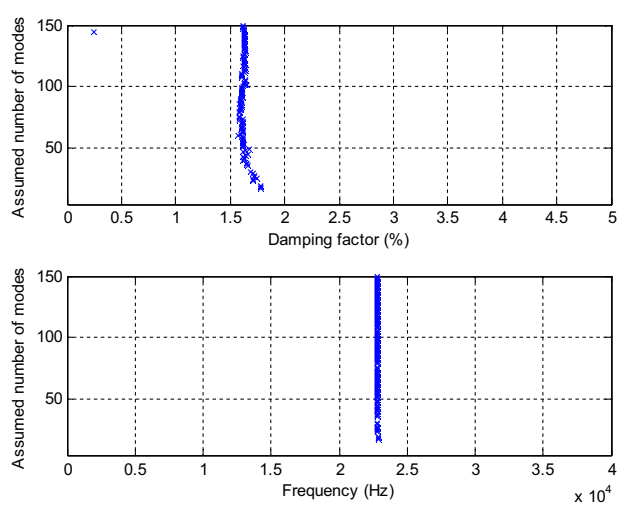

Figure 9. Stabilization diagram eigenfrequencies and damping factors for the perforated microplate.

Table 2. Parameters identified for the perforated microplate.

\begin{tabular}{|l|c|c|c|}
\hline $\begin{array}{l}\text { Resonance } \\
\text { frequency } F\end{array}$ & $\begin{array}{c}\text { Damping } \\
\text { factor } \xi\end{array}$ & $\begin{array}{c}\text { Stiffness } \\
\text { coefficient } k\end{array}$ & $\begin{array}{c}\text { Damping } \\
\text { coefficient } c\end{array}$ \\
\hline $22810 \mathrm{~Hz}$ & $1.62 \%$ & $78.33 \mathrm{~N}^{-1} \mathrm{~m}^{-1}$ & $17.71 \times 10^{-6}$ N.s.m \\
\hline
\end{tabular}

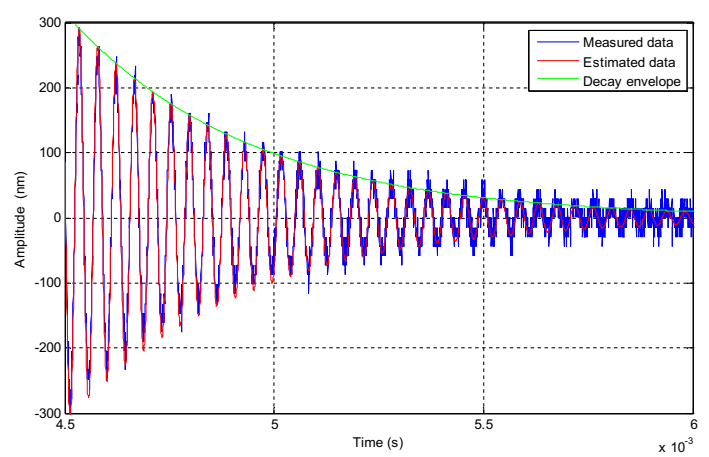

Figure 10. Comparison between the measured time response (in blue) and the identified time response (in red).

The decay function of the response is expressed by the relation $x(t)=A \exp (-D t)$, where $A$ is obtained by initial conditions and $D=2 \pi F \xi$ is computed from the identified modal parameters. This decay function is plotted in Fig. 10. Note that the coefficients $A$ and $D=$ $c /(2 \mathrm{~m})$ can also be evaluated directly by interpolating the measured response of the microplate and in this case we can obtain the value of the damping coefficient $c$ from $D$ and $m$.

\section{Conclusion}

The problem of modal parameter identification from output data only is very important because such parameters can be used for fault detection, structural health monitoring and model validation. We have proposed two methods for modal parameter identification, but we have proved that the shifted observability matrix method gives the same modal parameters than the shifted block row of the block Hankel matrix method. These relationships have been established in the exact data case and in the noisy data case. Numerical and experimental results have shown the effectiveness of subspace methods in modal parameter identification. 


\section{References}

[1] B.L. Ho and R.E. Kalman, "Regelungstechnik, 14, 3 (1966)

[2] S.Y. Kung. $12^{\text {th }}$ Asilomar Conference on Circuits and Systems, Asimolar, C.A. (1978)

[3] J.N. Juang Applied System Identification, Prentice Hall, New-Jersey, U.S.A. (1994)
[4] P. Van Overschee and B. de Moor Subspace identification for linear systems, Kluwer (1996)

[5] J. Lardiès and M.N Ta , Mechanical Systems and Signal Processing, 25, 17 (2011)

[6] G. De Pasquale and A. Soma, Proceedings of the $15^{\text {th }}$ DTIP, Cannes, France (2012) 